\title{
APPLICATIONS OF HOMOTOPY IN SHEAF THEORY
}

\author{
JAMES M. PARKS ${ }^{1}$
}

\begin{abstract}
Various mapping theorems for sheaf cohomology are obtained, where coefficients are in locally constant sheaves or limits of locally constant sheaves. Applications are made to the continuity of sheaf cohomology on homotopy-systems. A generalization of the Alexander-Čech cohomology continuity theorem is an immediate consequence.
\end{abstract}

1. Notation. The category of sheaves (of some algebraic structure) of a fixed (always Hausdorff) space $X$ will be denoted by $\mathfrak{A}_{X}$. The (full) subcategory of $\mathfrak{A}_{X}$ of locally constant sheaves (or limits of locally constant sheaves) on $X$ will be denoted by $\mathfrak{A}_{X}^{\prime}\left(\mathfrak{H}_{X}^{*}\right)$. For general definitions in sheaf theory see [1, Chapter 17] and [2]. All maps are assumed to be continuous.

The author wishes to thank his advisor, Professor D. G. Bourgin, for valuable conversations during the preparation of this material.

2. Applications in sheaf cohomology. If $f: X \rightarrow Y$ is a homotopy equivalence with homotopy inverse $g, \mathscr{A} \in \mathfrak{A}_{Y}^{\prime}$ with respect to the open cover $G$ of $Y$, and $F=f^{-1}(G)$, then $f$ is an $(F, G)$-homotopy equivalence (relative to $\mathscr{A}$ ) iff $f g \simeq 1_{Y}$ by a $G$-homotopy and $g f \simeq 1_{X}$ by a $F$-homotopy.

THEOREM 1. If $\mathscr{A} \in \mathfrak{A}_{Y}^{\prime}$ with respect to the cover $G$ of $Y, f: X \rightarrow Y$ is an $(F, G)$-homotopy equivalence, and $X$ and $Y$ are compact, then there exists a sheaf $\mathscr{B} \in \mathfrak{U}_{X}^{\prime}$ such that $H_{c}^{*}(X, \mathscr{B}) \approx H_{c}^{*}(Y, \mathscr{A})$.

Let $\mathscr{B}=f^{*} \mathscr{A}$ and $\left(f, f^{*}\right): S(Y, \mathscr{A}) \rightarrow S(X, \mathscr{B})$ be the homomorphism defined by $\left(f, f^{*}\right)(s)(x)=f_{x}^{*} s f(x)$, for all $s \in S(Y, \mathscr{A})$, where $\left\{f_{x}^{*}\right\}$ is the inverse image cohomomorphism.

Likewise, if $g$ is the homotopy inverse of $f$, define a homomorphism $\left(g, g^{*}\right): S(X, \mathscr{B}) \rightarrow S(Y, \mathscr{A})$. This map is well defined since locally constant

Received by the editors August 3, 1971.

AMS 1970 subject classifications. Primary 55B30; Secondary 55D99.

Key words and phrases. Locally constant sheaves, sheaf theory, limits of sheaves, continuity of sheaf cohomology, homotopy-systems, mapping theorems in sheaf cohomology.

${ }^{1}$ The author was supported by an NDEA IV Fellowship at the University of Houston during the preparation of parts of this material. Parts of this material appeared in the author's dissertation at the University of Houston.

(c) American Mathematical Society 1972 
sheaves behave like locally constant bundles with respect to homotopic maps of the base space ([5, p. 53], [4]). Thus $g^{*} f^{*} \mathscr{A} \approx \mathscr{A}$ and $f^{*} g^{*} f^{*} \mathscr{A} \approx$ $f^{*} \mathscr{A}$.

The map $\left(f, f^{*}\right)$ in onto, since $\operatorname{Im} f \cap U \neq \varnothing$ for all $U \in G$ by the $(F, G)$-homotopy equivalence property of $f$ (similarly for $\left(g, g^{*}\right)$ ). Note that stalkwise the maps are isomorphisms. Thus $S\left(X, f^{*} \mathscr{A}\right) \approx S(Y, \mathscr{A})$.

Similarly, for serrations [1, p. 481], $S\left(X, \mathscr{T}^{0} f^{*} \mathscr{A}\right) \approx S\left(Y, \mathscr{T}^{0} \mathscr{A}\right)$, and by iteration $S\left(X, \mathscr{T}^{*} f^{*} \mathscr{A}\right) \approx S\left(Y, \mathscr{T}^{*} \mathscr{A}\right)$, or $H_{c}^{*}\left(X, f^{*} \mathscr{A}\right) \approx H_{c}^{*}(Y, \mathscr{A})$.

An immediate application of Theorem 1 to sheaves in $\mathfrak{2}_{Y}^{*}$ is possible under somewhat restrictive assumptions.

CoROllary 2. Let $\left\{Y_{\alpha}, q_{\alpha}^{\beta}\right\}_{\Lambda}$ be an inverse system of compact spaces, $\left\{\mathscr{A}_{\alpha}, \Phi_{\beta}^{\alpha}\right\}_{\Lambda}$ a direct system of locally constant sheaves on $\left\{Y_{\alpha}, \varphi_{\alpha}^{\beta}\right\}_{\Lambda}$ with respect to covers $\left\{G_{\alpha}\right\}_{\Lambda}$, and let $\left\{X_{\alpha}, \psi_{\alpha}^{\beta}\right\}_{\Lambda}$ be an inverse system of compact spaces with $F=\left\{f_{\alpha}: X_{\alpha} \rightarrow Y_{\alpha} \mid f_{\alpha}\right.$ is an $\left(F_{\alpha}, G_{\alpha}\right)$-homotopy equivalence $\}$ a map of the systems. Let $Y=$ proj $\lim Y_{\alpha}, X=\operatorname{proj} \lim X_{\alpha}$, and $\mathscr{A}=\operatorname{inj} \lim \varphi_{\alpha}^{*} \mathscr{A}_{\alpha}$ (where $\varphi_{\alpha}: X \rightarrow X_{\alpha}$ ). Then there exists a sheaf $\mathscr{B} \in \mathfrak{A}_{X}^{*}$ such that $H_{c}^{*}(X, \mathscr{B}) \approx H_{c}^{*}(Y, \mathscr{A})$.

By Theorem 1 one has $H_{c}^{*}\left(X_{\alpha}, f_{\alpha}^{*} \mathscr{A}_{\alpha}\right) \approx H_{c}^{*}\left(Y_{\alpha}, \mathscr{A}_{\alpha}\right)$ for all $\alpha \in \Lambda$. By continuity [2, pp. 69-72], inj $\lim H_{c}^{*}\left(Y_{\alpha}, \mathscr{A}_{\alpha}\right) \approx H_{c}^{*}(Y, \mathscr{A})$ and inj $\lim H_{c}^{*}\left(X_{\alpha}, f_{\alpha}^{*} \mathscr{A}_{\alpha}\right) \approx H_{c}^{*}\left(X, f^{*} \mathscr{A}\right)$, where $f^{*} \mathscr{A}=\operatorname{inj} \lim \psi_{\alpha}^{*} f_{\alpha}^{*} \mathscr{A}_{\alpha} \approx$ inj $\lim f^{*} \varphi_{\alpha}^{*} \mathscr{A}_{\alpha}$. Let $\mathscr{B}=f^{*} \mathscr{A}$ and the result follows.

An existence theorem which satisfies the hypothesis of Corollary 2 for sheaves in $\mathfrak{A}_{Y}^{*}$ is established by the following theorem.

THEOREM 3. Let $\mathscr{A} \in \mathfrak{A}_{Y}^{*}, f: X \rightarrow Y$ be an $\left(F_{\alpha}, G_{\alpha}\right)$-homotopy equivalence for all $\alpha \in \Lambda$, where $\mathscr{A}=\operatorname{inj} \lim \mathscr{A}_{\alpha}$. Suppose $X$ and $Y$ are compact spaces. Then there exists a sheaf $\mathscr{B} \in \mathfrak{H}_{X}$ such that $H_{c}^{*}(Y, \mathscr{A}) \approx H_{c}^{*}(X, \mathscr{B})$.

Imbed $Y$ in $\prod_{\alpha \in \Lambda} I_{\alpha}$, where $\Lambda=I^{Y}$, and construct an inverse system of finite polyhedra $\left\{Y_{\alpha}, \varphi_{\alpha}^{\beta}\right\}_{\Lambda}$ such that $Y=\operatorname{proj} \lim Y_{\alpha}$ [3, p. 284].

Let $Z_{\varphi_{\alpha}}$ be the mapping cylinder of the projection map $\varphi_{\alpha}: Y \rightarrow Y_{\alpha}$ (recall $Z_{\varphi_{\alpha}} \simeq Y_{\alpha}$ ). Thus $\left\{Z_{\varphi_{\alpha}}, \tilde{\varphi}_{\alpha}^{\beta}\right\}_{\Lambda}$ is an inverse system, where $\tilde{\varphi}_{\alpha}^{\beta}$ is induced by $\varphi_{\alpha}^{\beta}$, and proj $\lim Z_{\varphi_{\alpha}}=Y \times I \simeq Y$.

Let $\mathscr{A}_{\alpha} \times[0,1)$ be the sheaf on $Y \times[0,1)$ such that $\left(\mathscr{A}_{\alpha} \times[0,1)\right)_{(x, t)}=$ $\left(\mathscr{A}_{\alpha}\right)_{x}$ for all $(x, t) \in Y \times[0,1)$. Since $Y \times[0,1)$ is locally closed in $Z_{\varphi_{\alpha}}$, extend $\mathscr{A}_{\alpha} \times[0,1)$ to $Z_{\varphi_{\alpha}}$ by zero and denote this sheaf by $\tilde{\mathscr{A}}_{\alpha}$. Thus inj $\lim \tilde{\mathscr{A}}_{\alpha}=\tilde{\mathscr{A}}=(\mathscr{A} \times[0,1)) \cup \theta$.

Carry out a similar construction on $X$ and the system $\left\{f^{*} \mathscr{A}_{\alpha}\right\}$.

Clearly, $H_{c}^{*}(Y, \mathscr{A}) \approx H_{c}^{*}(Y \times[0,1), \mathscr{A} \times[0,1)) \approx H_{c}^{*}(Y \times I, \mathscr{A})$. Similarly for $X$ and $\left(f^{*} \mathscr{A}_{\alpha}\right)^{\sim}=\operatorname{inj} \lim \tilde{f}^{*} \tilde{\mathscr{A}}_{\alpha}$. 
By continuity [2, pp. 69-72],

and

$$
H_{c}^{*}(Y, \mathscr{A}) \approx H_{c}^{*}(Y \times I, \tilde{\mathscr{A}}) \approx \operatorname{inj} \lim H_{c}^{*}\left(Z_{\varphi_{\alpha}}, \tilde{\mathscr{A}}_{\alpha}\right),
$$

$$
H_{c}^{*}\left(X, f^{*} \mathscr{A}\right) \approx H_{c}^{*}\left(X \times I,\left(f^{*} \mathscr{A}\right)^{\sim}\right) \approx \operatorname{inj} \lim H_{c}^{*}\left(Z_{\psi_{x}}, \tilde{f}^{*} \tilde{\mathscr{A}}_{\alpha}\right)
$$

where $f$ is the extension of $f$ to $Z_{\psi_{\alpha}}$.

By Theorem $1, H_{c}^{*}\left(Z_{\varphi_{\alpha}}, \tilde{\mathscr{A}}_{\alpha}\right) \approx H_{c}^{*}\left(Z_{\varphi_{\alpha}}, \tilde{f}^{*} \tilde{\mathscr{A}}_{\alpha}\right)$ for all $\alpha \in \Lambda$, since $f$ is an $\left(F_{\alpha}, G_{\alpha}\right)$-homotopy equivalence (and thus $\tilde{f}$ is an $\left(\tilde{F}_{\alpha}, \tilde{G}_{\alpha}\right)$-homotopy equivalence, where $\tilde{G}_{\alpha}$ is the cover $\left.G_{\alpha} \times[0,1) \cup Y_{\alpha}\right)$. Thus $H_{c}^{*}(Y, \mathscr{A}) \approx$ $H_{c}^{*}\left(X, f^{*} \mathscr{A}\right)$, and $\mathscr{B}=f^{*} \mathscr{A}$.

3. Continuity theorems. The introduction of homotopy-systems in the above setting precipitates the following results on continuity (see [4] for basic definitions and properties of homotopy-systems).

An inverse system of spaces $\left\{X_{\alpha}, q_{\alpha}^{\beta}\right\}_{\Lambda}$ is called a homotopy-inverse system ( $F_{\alpha}$-homotopy-inverse system) iff whenever $\alpha, \beta, \gamma \in \Lambda$ and $\alpha<\beta<\gamma$, then $\varphi_{\alpha}^{\beta} \varphi_{\beta}^{\gamma} \simeq \varphi_{\alpha}^{\gamma}\left(\varphi_{\alpha}^{\beta} \varphi_{\beta}^{\gamma} \simeq \varphi_{\alpha}^{\gamma}\right.$ by a $F_{\alpha}$-homotopy for some cover $F_{\alpha}$ of $\left.X_{\alpha}\right)$.

THeOREM 4. If $\left\{X_{\alpha}, \varphi_{\alpha}^{\beta}\right\}_{\Lambda}$ is a $F_{\alpha}$-homotopy-inverse system of locally path connected compact spaces with respect to covers $\left\{F_{\alpha}\right\}_{\Lambda}$ determined by some system $\left\{\mathscr{A}_{\alpha}, \Phi_{\beta}^{\alpha}\right\}_{\Lambda}$ of locally constant sheaves on $\left\{X_{\alpha}, \varphi_{\alpha}^{\beta}\right\}_{\Lambda}$ (where $\Phi_{\beta}^{\alpha}$ are $\varphi_{\alpha}^{\beta}$-cohomorphisms $)$, then $H_{c}^{*}(X, \mathscr{A})=H_{c}^{*}\left(\right.$ h-proj $\left.\lim X_{\alpha}, \operatorname{inj} \lim \varphi_{\alpha}^{*} \mathscr{A}_{\alpha}\right) \approx$ inj $\lim H_{c}^{*}\left(X_{\alpha}, \mathscr{A}_{\alpha}\right)$.

The homotopy-inverse limit space, $X=\mathrm{h}$-proj $\lim X_{\alpha}$, is compact [4], and if $\varphi_{\alpha}: X \rightarrow X_{\alpha}$ is the projection map, then $\varphi_{\alpha}^{*} \mathscr{A}_{\alpha}$ is a locally constant sheaf on $X$ with respect to the cover $\varphi_{\alpha}^{-1}\left(F_{\alpha}\right)$. If $\mathscr{A}=$ inj $\lim \varphi_{\alpha}^{*} \mathscr{A}_{\alpha}$, then inj $\lim H_{c}^{*}\left(X, \varphi_{\alpha}^{*} \mathscr{A}_{\alpha}\right) \approx H_{c}^{*}(X, \mathscr{A})$. In view of the fact that the sheaves $\left\{\mathscr{A}_{\alpha}\right\}$ and $\left\{\varphi_{\alpha}^{*} \mathscr{A}_{\alpha}\right\}$ are locally constant, the behavior of the direct limit functor on $\left\{H_{c}^{*}\left(X_{\alpha}, \mathscr{A}_{\alpha}\right)\right\}$ is identical to the usual situation and the result follows.

The following corollary is immediate.

COROllary 5. If $X$ is compact and $\mathscr{A}$ is the limit of sheaves in $\mathfrak{A}_{X}^{*}$, then $H_{c}^{*}(X, \mathscr{A})$ may be expressed as a doubly iterated limit of cohomologies of spaces of the homotopy type of polyhedra with coefficients in locally constant sheaves.

If constant sheaves are present in Theorem 4 the $F_{\alpha}$-homotopy condition in Theorem 4 may be weakened to give the following generalization of the Alexander-Čech continuity theorem [1, p. 168].

COROLlaRY 6. If $\left\{X_{\alpha}, \varphi_{\alpha}^{\beta}\right\}_{\Lambda}$ is a homotopy-inverse system of locally path connected compact spaces, then $H_{c}^{*}\left(\mathrm{~h}-\right.$ proj $\left.\lim X_{\alpha}, R\right) \approx \operatorname{inj} \lim H_{c}^{*}\left(X_{\alpha}, R\right)$.

An analogous result may be obtained for Čech homology. 


\section{REFERENCES}

1. D. G. Bourgin, Modern algebraic topology, Macmillan, New York, 1963. MR 28 \#3415.

2. Glen E. Bredon, Sheaf theory, McGraw-Hill, New York, 1967. MR 36 \#4552.

3. S. Eilenberg and N. E. Steenrod, Foundations of algebraic topology, Princeton Univ. Press, Princeton, N.J., 1952. MR 14, 398.

4. James M. Parks, Homotopy-systems, H-spaces and sheaf cohomology, Ph.D. Dissertation, University of Houston, Houston, Texas, 1971.

5. N. E. Steenrod, The topology of fibre bundles, Princeton Math. Series, vol. 14, Princeton Univ. Press, Princeton, N.J., 1951. MR 12, 522.

Department of Mathematics, University of Houston, Houston, Texas 77004 